\title{
RECOGNITION OF ANHYDRITE INTERCALATED SALT DEPOSIT FROM SEISMIC DATASET DISTORTED BY NOISE
}

\author{
EWA KAWALEC-LATAŁA \\ AGH University of Science and Technology, Faculty of Geology, Geophysics and Environment Protection, Krakow, Poland. \\ E-mail: ewal@geol.agh.edu.pl
}

\begin{abstract}
Acoustic inversion is useful to extract information from seismic data. Inhomogeneities of salt deposits should be predicted before the decision of underground storage location is made. The work concerns the possibility of detecting anhydrite intercalation in the rock salt from seismic dataset. The resolution strongly depends on signal to noise ratio. The synthetic pseudoacoustic impedance sections are generated for efficiency test of predictive and minimum entropy deconvolution process, when random noise distorts the seismic traces.
\end{abstract}

Key words: underground storage, acoustic impedance, salt deposit, inhomogeneity detection, noise

\section{INTRODUCTION}

Rock mass containing salt deposits displays high seismologic inhomogeneity. Both geometrical and lithological inhomogeneities of salt deposits must be predicted before the underground storage location is fixed. Geometrical inhomogeneities are related to thickness changes, dying-out of strata [3], etc. Lithological inhomogeneities are related to the presence of different kinds of salts and facial changes. Many technical problems are connected with intercalation of anhydrite within rock salt beds [7]. A reflection seismic method forms a vast data source of information on the structure of the subsurface. The reflection seismic method creates a geophysical sampled picture of the subsurface, which has to be interpreted in a geologically meaningful manner.

The interface between layers is usually related to sedimentary bedding plane. The seismic response of reflected wave is dependent on the amount of acoustic impedance (AI) changes over the interface. Acoustic impedance is defined as the multiplication of density and velocity $\mathrm{AI}=$ density $\times$ velocity.

Each seismic layer in the subsurface has its own acoustic impedance. The seismic section displayed an amplitude which is proportional to the reflection strength. The reflection coefficient is a function of acoustic impedance.

Acoustic inversion AI transforms the seismic section to pseudoacoustic impedance section. Inversion of seismic data is widely used in investigation of hydrocarbons to estimate reservoir geometry and petrophysical feature. High resolution pseudoacoustic impedance sections allow detailed lithology-fluid prediction at various stages of the reservoir life cycle [8]. The benefit of the inversion is that the seismic resolution is increased and pseudoacoustic impedance is closely connected to acoustic impedance of layers, so geological interpretation is more comfortable. There are several ways to achieve such transformation [11]. The acoustic inversion method is useful for detection of inhomogeneities, but in practice is complicated and in some cases visual interpretation is difficult. The nonuniqueness in the seismic inversion is mainly due to band limited frequency even for noise free data [1], [9]. In practice, forward-modelling simplification is needed to estimate the resolution in reasonable time; especially, for adaptation of acoustic inversion AI method to a new target as recognition of inhomogeneities of salt deposits.

Signal parameters briefly influenced the resolution. The resolution is controlled by spectral bandwidth [4]. For vertical resolution the yardstick is the dominant wavelength, where wave velocity is divided by dominant frequency. The vertical resolution is understood as a limit on two reflections being still separable, one from bottom one from the top.

In practice, seismic traces, are always distorted by random noise and wave propagation phenomena. The random noise is independent of time. This means that the level of random noise is constant throughout the 
registration time. When the noise in seismic data decreases the accuracy and reliability of interpretation increases [2]. Unfortunately, complete elimination of noise from seismic measurements is not possible When the signal to noise ratio N/S is poor, visual interpretation is especially difficult or sometimes impossible [2]. Modern seismic data processing can eliminate much of the distortion. An important procedure to help repair the damage to the spectrum (caused by seismic source, transmission through the earth and the recording instruments) is a deconvolution [10]. The inversion procedure is not a unique process. This nonuniqueness is mainly due to band limited frequency even for noise free data [1], [9]. So, modelling pseudoacoustic impedance sections is recommended for verification of the effectiveness of deconvolution process for the inversion procedure and, as a result, for accuracy and reliability interpretation.

The work concerns the possibility of recognizing space distribution of anhydrites in the rock salt from seismic data by interpretation of pseudoimpedance acoustic sections obtained by acoustic inversion AI. The effectiveness of predictive deconvolution and minimum entropy deconvolution MED is discussed for the data strongly distorted by the random noise. Numerous examples of modelling are presented. The synthetic sections are constructed for high noise level $(20 \%)$.

The seismic traces are generated for seismogeological model. A simplified seismological model is created on the basis of geological data from LGOM area [6], [7]

\section{MODELLING}

For the entire process, the authors' own computer system INVERS was used [5]. The system INVERS consists of two main parts: (1) modelling, (2) visualization of results.

First, the synthetic seismic traces are generated for seismogeological model. Synthetic seismogram was calculated as a convolution of reflection coefficient series with seismic signal. The signal of Puzyriev $s(t)$ was used

$$
s(t)=\exp \left(-\beta^{2} \cdot t^{2}\right) \cdot \sin \left(2 \pi f_{0} t \pm \varphi_{0}\right) .
$$

The parameters of the signal are: $f_{0}$ - dominant frequency, $\varphi_{0}$ - initial phase, $\beta$-dumping factor.

The ratio $f_{0} / \beta$ determines the length of signal. When the ratio $f_{0} / \beta=1$ the signal is relatively short, the ratio $f_{0} / \beta=2$ gives the long signal. Next, seismic traces were distorted by the random noise.

The seismic trace is defined as convolution of the reflection coefficient series and source wavelet. Synthetic seismogram with additive noise has the form

$$
x(t)=r(t) * w(t)+n(t)
$$

where $r(t)$ - the reflection coefficient series, $w(t)-$ source wavelet, $x(t)$ - synthetic trace, $n(t)$ - additive random noise. Following the simulation of the seismic sections the acoustic inversion AI was done.

The acoustic inversion of seismic traces makes its possible to obtain pseudoacoustic impedance traces. The resolution of the pseudoacoustic impedance sections depends in reality on signal parameters and noise level. For band limited seismic data an important procedure to higher resolution is a deconvolution.

\section{DECONVOLUTION OF SEISMIC TRACES}

In a mathematical sense one must find the solution to an equation form (2).

Reflectivity function $r(t)$ is the required solution.

Inversion process used the seismic trace as the reflection coefficient series. In practice, the seismic trace is only an approximation additionally distorted by noise. The deconvolved trace gives an approximation to the required reflection coefficients. The quality of the approximation is dependent upon the applicability of the basic assumptions. The deconvolution is an effective tool for improvement of digital inversion [10].

The classical procedure of decolvolution such as predictive or spiking deconvolution [10] attempt to whiten the spectra. The minimum entropy deconvolution MED [12] approach seeks a solution consisting mainly of isolated spikes, that is, the smallest number of large spikes there are consistent with the data. The minimum entropy deconvolution MED is a technique proposed by Wiggins with the purpose of separating the components of the signal, as the convolution model of smooth wavelet with a series of impulses. The minimum entropy deconvolution MED concerns the maximization Varimax norm

$$
V(\mathbf{r})=\sum_{i}\left(\frac{r_{i}}{\|\mathbf{r}\|}\right)^{4}
$$

where $\mathbf{r}$ is the reflection coefficient sequence vector.

Minimum entropy deconvolution MED provide for obtaining the smallest number of large spikes in the 
data. The inversion procedure is not a unique process, so the modelling of pseudoimpedance acoustic sections is useful for the evaluation of the influence of noise on the effective deconvolutions for resolution.

\section{RESULTS - MODELLING EXAMPLE}

Pseudoacoustic impedance sections for different signal parameters and 20\% random noise level were constructed. The seismic traces are generated for seismogeological model. A simplified seismological model consists of geometrical definitions of the rock salt bed intercalated by thin anhydrite layers dying out and velocity defined elastic feather of rocks [6]. A theoretical, simplified model is presented in Fig. 1.
In the figure, the left vertical axis is the depth in meters, horizontal axis: number of traces, right axis is the wave velocity in $\mathrm{m} / \mathrm{s}$ displayed as colour scale. The horizontal scale is the distance on surface along seismic profile. The top of the salt rock is at $300 \mathrm{~m}$ and the bottom at about $460 \mathrm{~m}$. The model shows the thickness changes due to dying-out of strata of anhydrite. The thickness of anhydrite changes from 80 to $0 \mathrm{~m}$ on the left side, and from 30 to $0 \mathrm{~m}$ on the right side, Fig. 1. A synthetic impulse seismogram was calculated on the basis of this model. Next the seismic synthetic seismograms for different parameters were generated. For this purpose, the impulse seismogram was convolved with various seismic signals. For long and short signals for the same dominant frequency of $40 \mathrm{~Hz}$. The synthetic seismograms were free from noise or

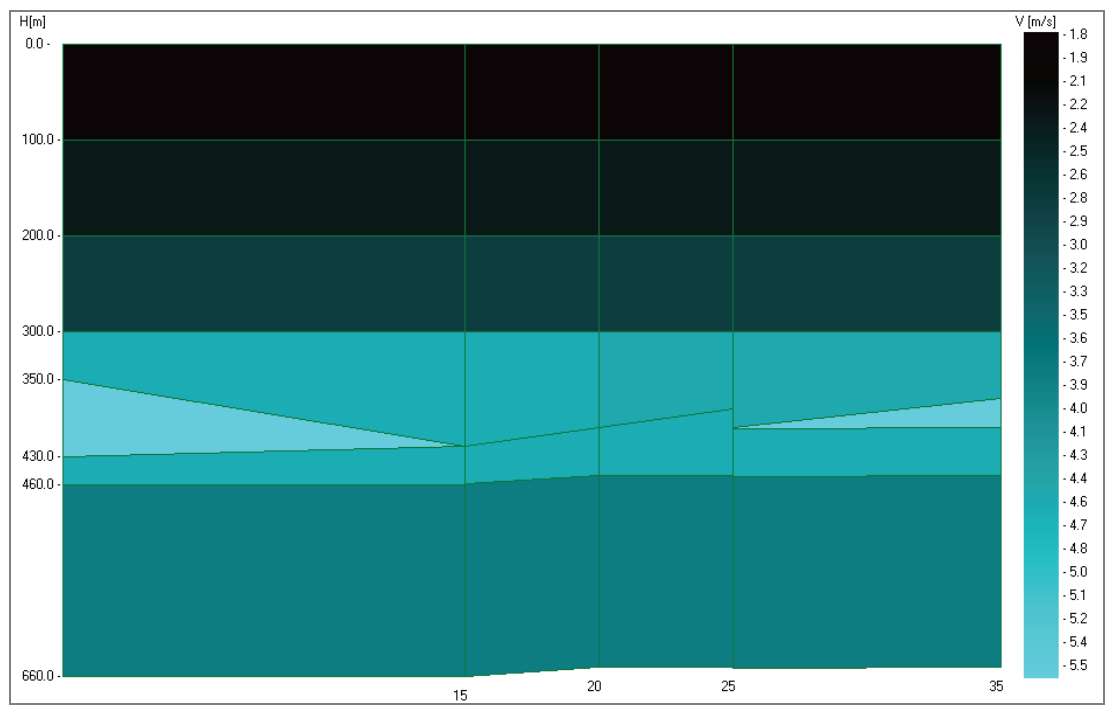

Fig. 1. Simplified seismological model

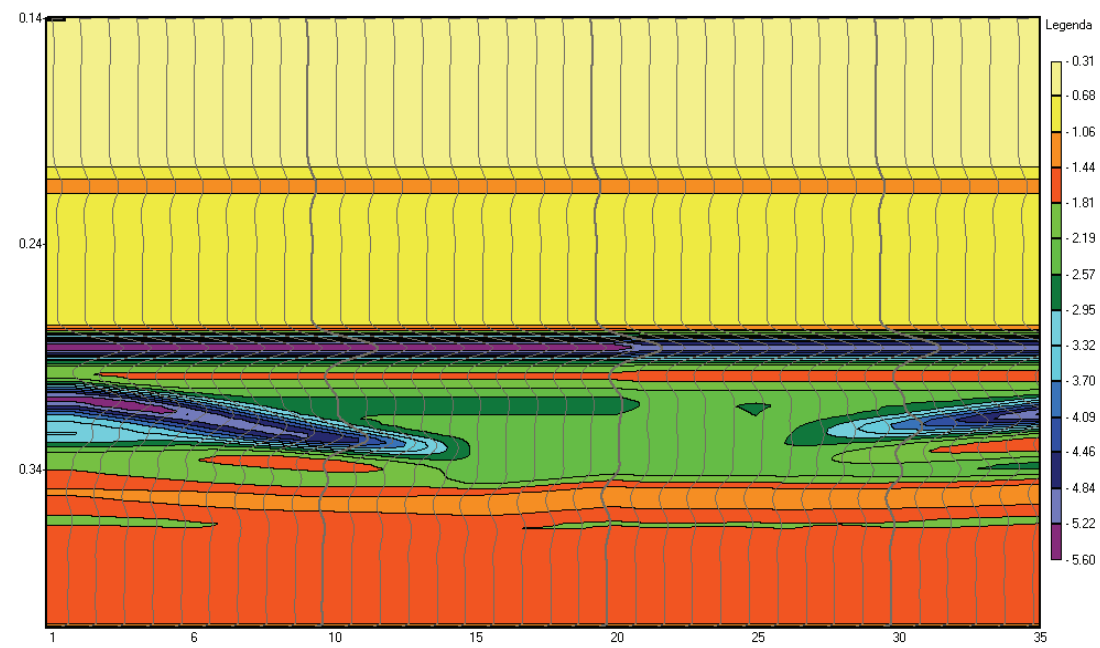

Fig. 2. Synthetic pseudoimpedance acoustic section; signal parameters:

dominant frequency $f_{0}=40 \mathrm{~Hz}$, initial phase $\varphi_{0}=0$, the ratio of dominant frequency to dumping factor $f_{0} / \beta=1$, noise $0 \%$ 


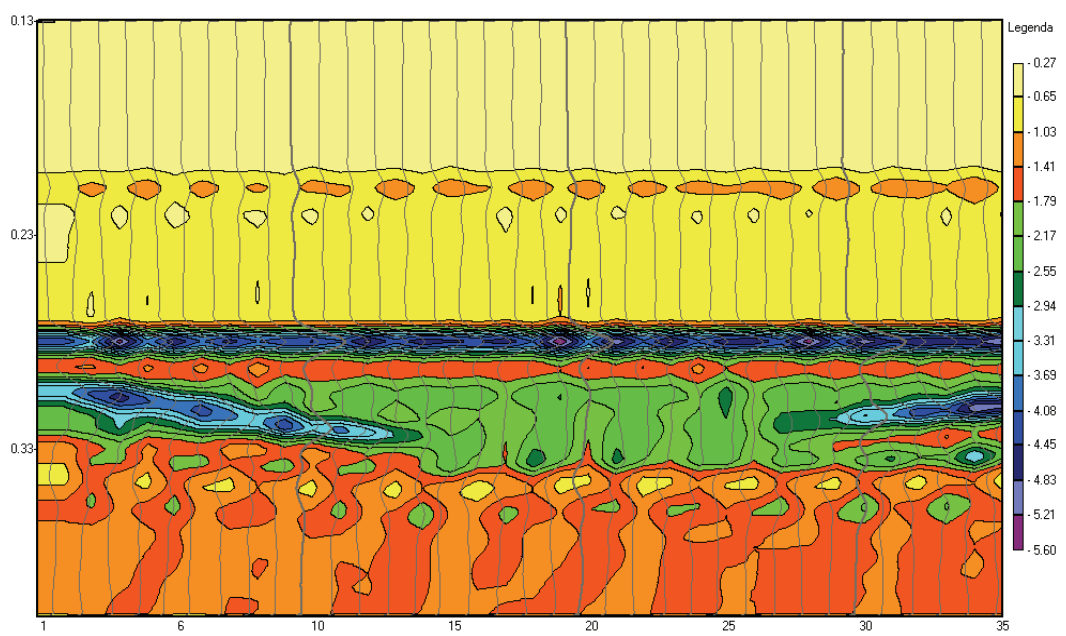

Fig. 3. Synthetic pseudoimpedance acoustic section; signal parameters: dominant frequency $f_{0}=40 \mathrm{~Hz}$, initial phase $\varphi_{0}=0$,

the ratio of dominant frequency to dumping factor $f_{0} / \beta=1$, noise $20 \%$

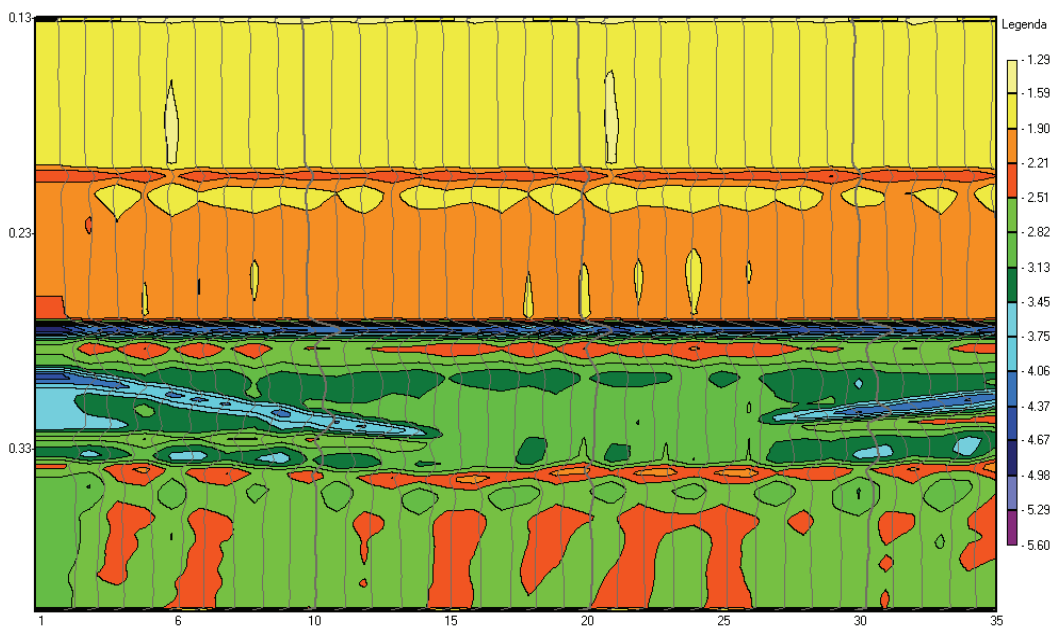

Fig. 4. Synthetic pseudoimpedance acoustic section with predictive deconvolution; signal parameters: dominant frequency $f_{0}=40 \mathrm{~Hz}$, initial phase $\varphi_{0}=0$,

the ratio of dominant frequency to dumping factor $f_{0} / \beta=1$, noise $20 \%$

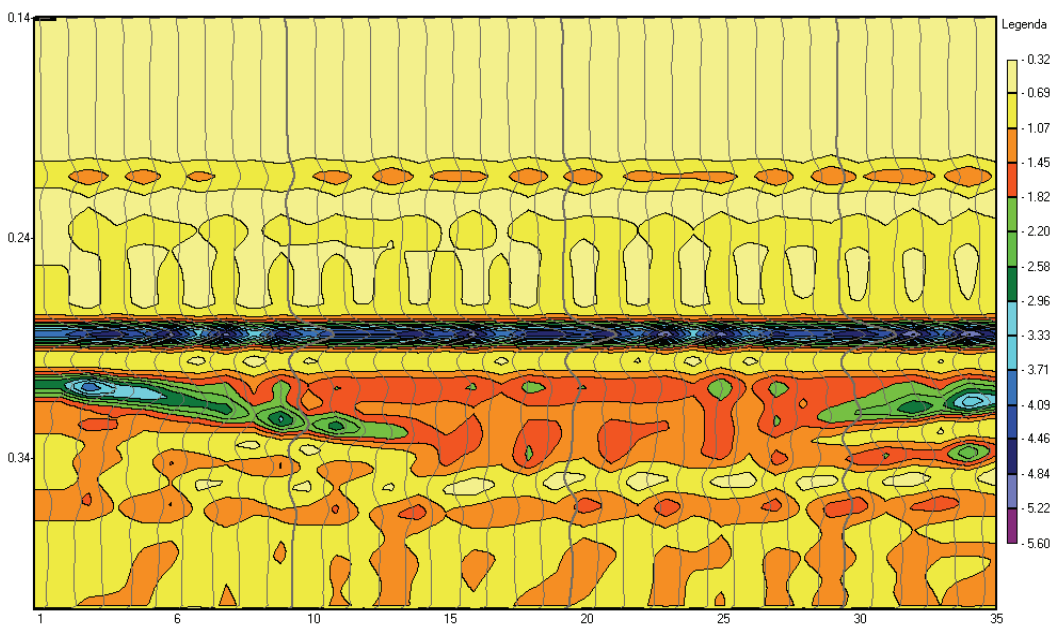

Fig. 5. Synthetic pseudoimpedance acoustic section with MED deconvolution; signal parameters: dominant frequency $f_{0}=40 \mathrm{~Hz}$, initial phase $\varphi_{0}=0$, the ratio of dominant frequency to dumping factor $f_{0} / \beta=1$, noise $20 \%$ 
a random $20 \%$ noise was added. The seismic sections thus constructed were the input data to acoustic inversion (AI). The process transforms seismic sections to pseudoimpedance acoustic sections. In some cases the deconvolution procedure was involved before. The pseudoimpedance acoustic sections generated for the dominant frequency $40 \mathrm{~Hz}$ and the ratio of dominant frequency to dumping factor $f_{0} / \beta=1$ (short signal) are presented in Figs. 2-5. In all the figures presenting pseudoimpedance sections the colour scale is reflecting the relative velocity (or acoustic impedance) variations. The left scale is the time [s]. The numbers of seismic traces on surface along seismic profile are on the horizontal scale.

The signal applied to construction of synthetic pseudoimpedance acoustic section is short, dominant frequency $f_{0}=40 \mathrm{~Hz}$ is low, noise is $0 \%$. The pseudoimpedance acoustic section has good resolution.

The data with random noise on the level $20 \%$ have rather low $S / N$ ratio. The presence of anhydrite is visible, but on the left side of pseudoimpedance acoustic section the anhydrite's layer thickness is not accurately mapped. Synthetic pseudoimpedance acoustic section generated with the same signal parameters as in Fig. 3, but with the process deconvolution included is presented in Figs. 4 and 5.

When predictive deconvolution procedure was applied before inversion the anhydrite's layer thickness on the left side of pseudoimpedance acoustic section is accurately mapped.

When minimum entropy deconvolution MED is applied before inversion, only the presence of anhydrite is visible on the pseudoimpedance acoustic sec-

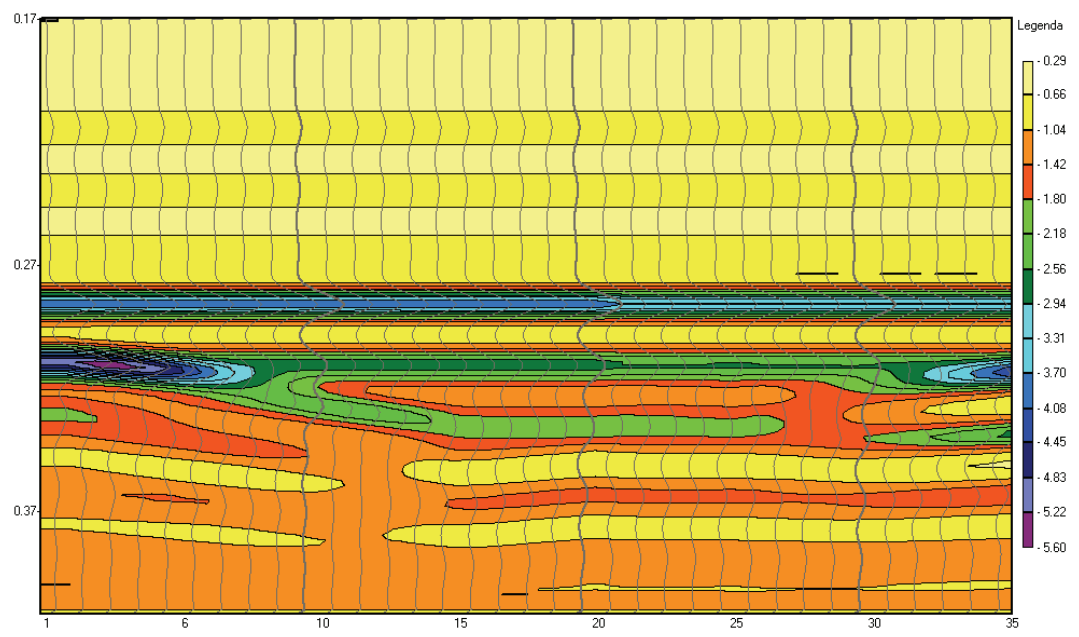

Fig. 6. Synthetic pseudoimpedance acoustic section; signal parameters: dominant frequency $f_{0}=40 \mathrm{~Hz}$, initial phase $\varphi_{0}=0$, the ratio of dominant frequency to dumping factor $f_{0} / \beta=2$, noise $0 \%$

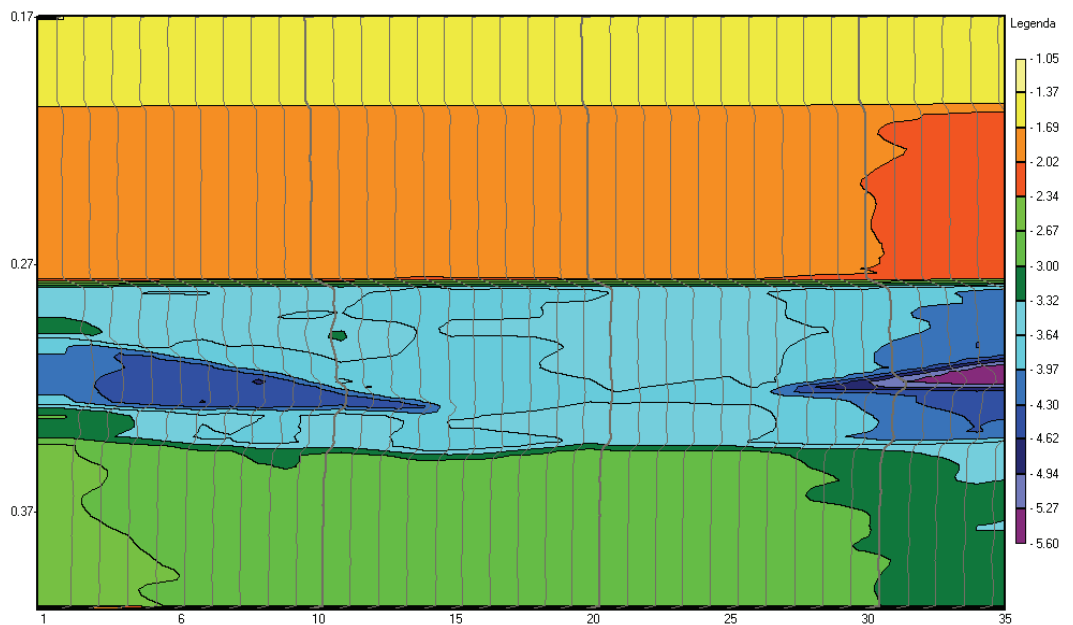

Fig. 7. Synthetic pseudoimpedance acoustic section with predictive deconvolution; signal parameters: dominant frequency $f_{0}=40 \mathrm{~Hz}$, initial phase $\varphi_{0}=0$, the ratio of dominant frequency to dumping factor $f_{0} / \beta=2$, noise $0 \%$ 


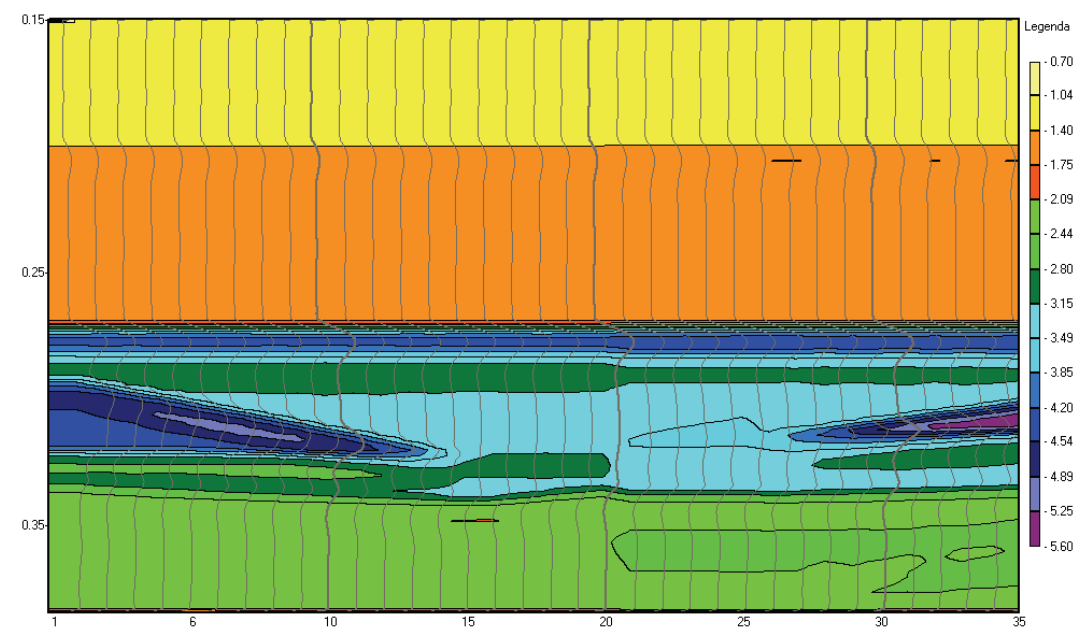

Fig. 8. Synthetic pseudoimpedance acoustic section with MED deconvolution; signal parameters: dominant frequency $f_{0}=40 \mathrm{~Hz}$, initial phase $\varphi_{0}=0$, the ratio of dominant frequency to dumping factor $f_{0} / \beta=2$, noise $0 \%$

tion but the anhydrite's layer thickness is not accurately mapped. The pseudoimpedance acoustic sections generated without noise for the dominant frequency $40 \mathrm{~Hz}$ and the ratio of dominant frequency to dumping factor $f_{0} / \beta=2$ (long signal) are presented in Figs. 6-8.

When the seismic signal is long, even for noisefree data the horizontal reach of the anhydrite's layer is not visible on the pseudoimpedance acoustic section.

When minimum entropy deconvolution MED or predictive deconvolution is applied before inversion the anhydrite's intercalation at both sides on the pseudoimpedance acoustic section is accurately mapped. The result is even better when minimum entropy deconvolution MED is applied. The data are without noise. Such a situation never occurs in reality. So, the examples show how much the deconvolution procedure is an effective tool in the case of long seismic signal.

The pseudoimpedance acoustic sections generated for the dominant frequency $40 \mathrm{~Hz}$ and the ratio of dominant frequency to dumping factor $f_{0} / \beta=2$ (long signal), with noise being added, are presented in Figs. 9-11.

When the pseudoimpedance acoustic section is generated for the dominant frequency $40 \mathrm{~Hz}$ and the long signal and the data are distorted by $20 \%$ noise the anhydrite's intercalation is visible but weakly mapped.

In the case of pseudoimpedance acoustic sections generated for low dominant frequency $40 \mathrm{~Hz}$ and long

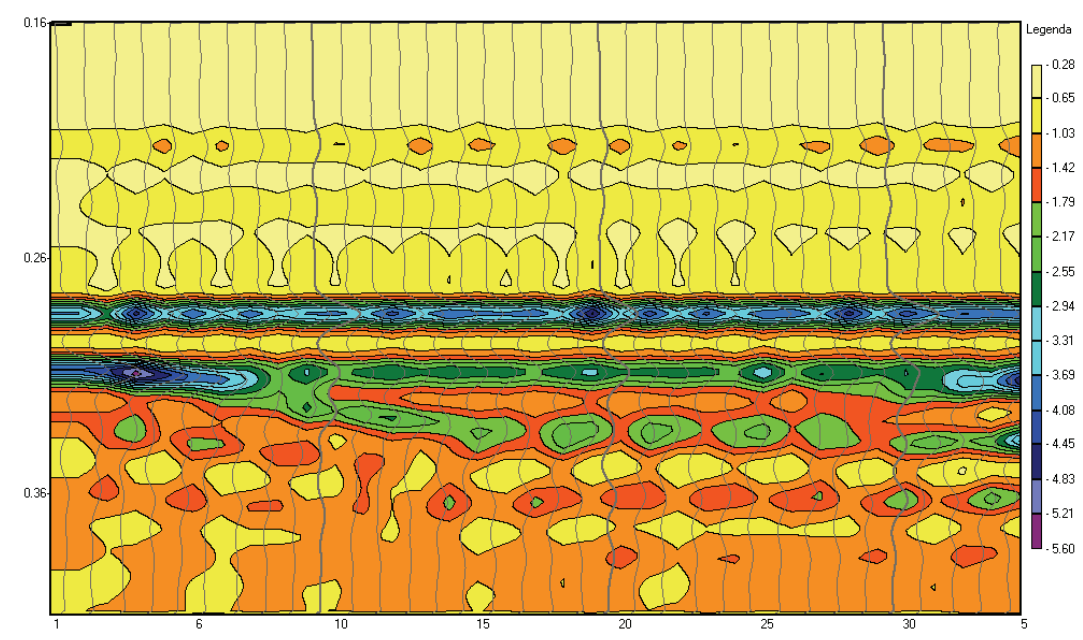

Fig. 9. Synthetic pseudoimpedance acoustic section; signal parameters: dominant frequency $f_{0}=40 \mathrm{~Hz}$, initial phase $\varphi_{0}=0$,

the ratio of dominant frequency to dumping factor $f_{0} / \beta=1$, noise $20 \%$ 


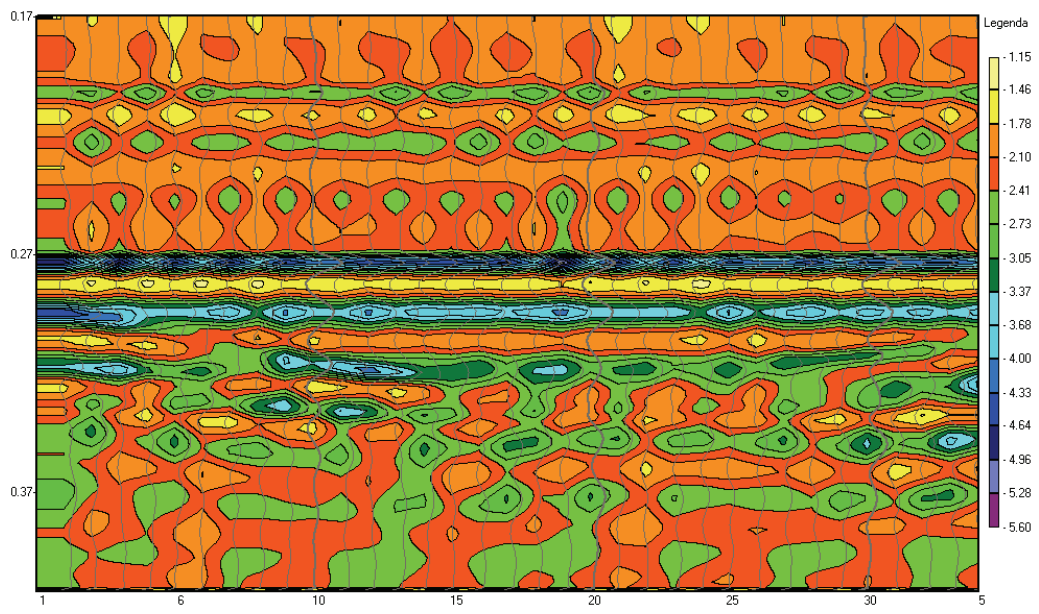

Fig. 10. Synthetic pseudoimpedance acoustic section with predictive deconvolution; signal parameters: dominant frequency $f_{0}=40 \mathrm{~Hz}$, initial phase $\varphi_{0}=0$,

the ratio of dominant frequency to dumping factor $f_{0} / \beta=1$, noise $0 \%$

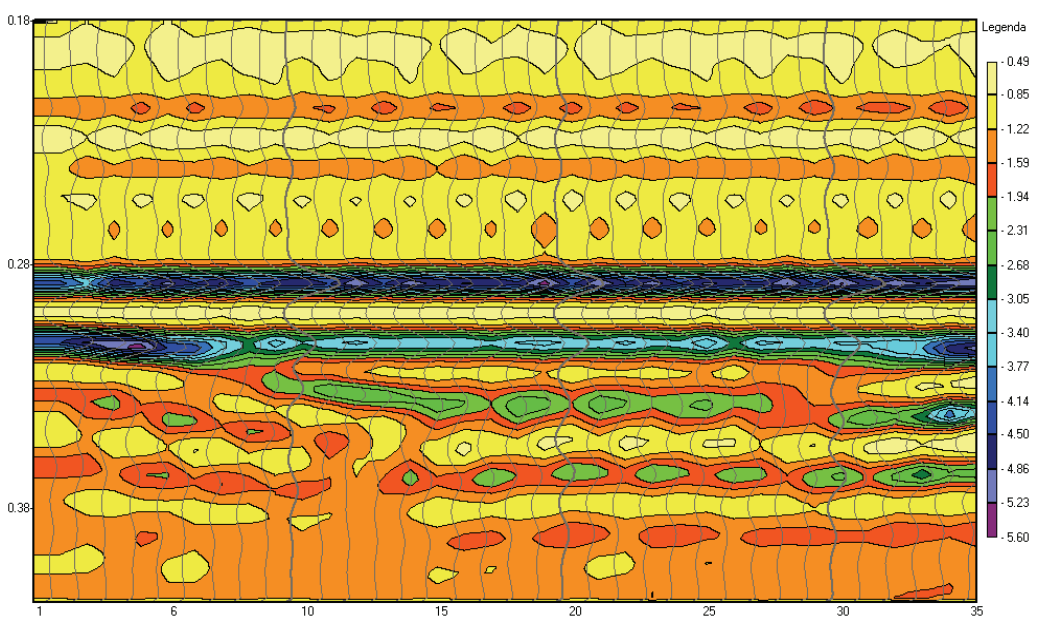

Fig.11. Synthetic pseudoimpedance acoustic section with MED deconvolution; signal parameters: dominant frequency $f_{0}=40 \mathrm{~Hz}$, initial phase $\varphi_{0}=0$,

the ratio of dominant frequency to dumping factor $f_{0} / \beta=1$, noise $0 \%$

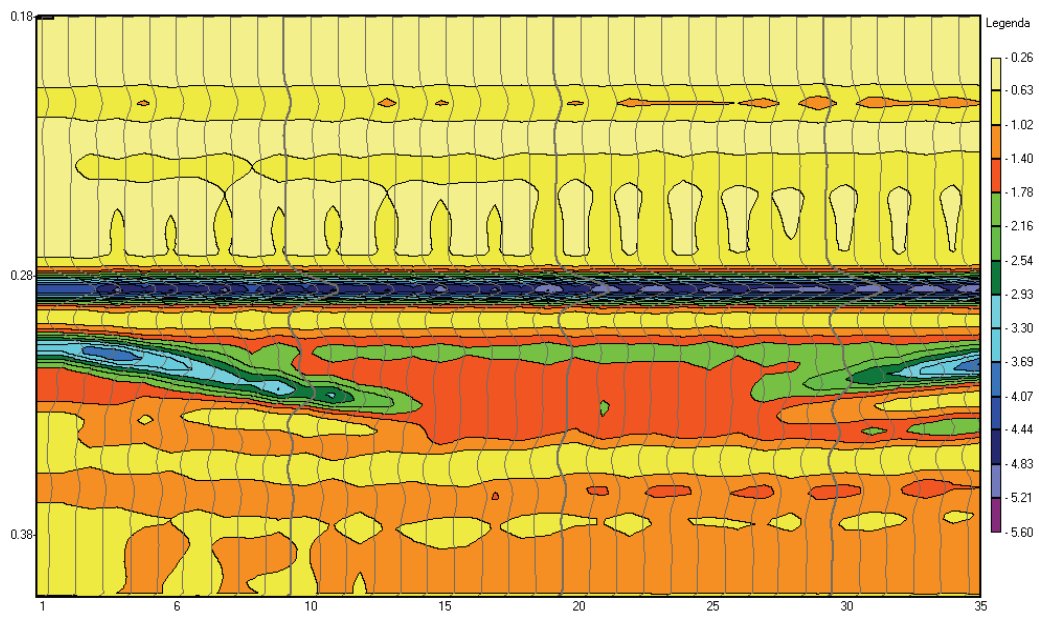

Fig. 12. Synthetic pseudoimpedance acoustic section with MED deconvolution; signal parameters: dominant frequency $f_{0}=40 \mathrm{~Hz}$, initial phase $\varphi_{0}=0$,

the ratio of dominant frequency to dumping factor $f_{0} / \beta=2$, noise $5 \%$ 
signal (the ratio of dominant frequency to dumping factor $f_{0} / \beta=2$ ) and high noise level with predictive deconvolution done before inversion the procedure is not effective. The result is unsatisfactory.

When the pseudoimpedance acoustic sections are generated for low dominant frequency $40 \mathrm{~Hz}$ and long signal (the ratio of dominant frequency to dumping factor $f_{0} / \beta=2$ ) and high noise level, with the minimum entropy deconvolution MED done before inversion, the resolution of the pseudoimpedance acoustic sections is slightly higher. The presence of anhydrite is visible, but the intercalation of anhydrite is weakly mapped.

When noise level is low 5\% the minimum entropy deconvolution MED done before inversion enhances the resolution. The result is presented in Fig. 12.

The resolution of the pseudoimpedance acoustic sections generated for low noise level even at the dominant frequency $40 \mathrm{~Hz}$ and long signal (the ratio of dominant frequency to dumping factor $f_{0} / \beta=2$ ) is clearly higher. The presence of anhydrite is visible, but the anhydrite's layer thickness is not accurately mapped. Relatively low noise increases efficiency of deconvolution.

\section{SUMMARY}

Inversion AI helps to extract additional information from seismic data. The process effectively transforms seismic into pseudoimpedance acoustic section. Such transformation means that seismic information is transformed into the geological domain. It facilities the interpretation. The synthetic pseudoimpedance acoustic sections presented here show how the deconvolution done before inversion processes in the presence of random noise improve its resolution. The data with random noise on a $20 \%$ level have rather low $S / N$ ratio. It is necessary to underline that when the sections are constructed for low signal to noise ratio $S / N$, especially when the model simulates high noise and poor signal parameters: low dominant frequency and long seismic signal (the ratio of dominant frequency to dumping factor $f_{0} / \beta=2$ ), the effect can be sometimes unsatisfactory. In many cases when minimum entropy deconvolution MED or predictive deconvolution is applied before inversion the anhydrite's intercalation on the pseudoimpedance acoustic section is accurately mapped. The result strongly depends on the level of noise. The resolution is slightly higher when minimum entropy deconvolution MED is applied. The presence of anhydrite is usually visible, but the anhydrite's intercalation is sometimes weakly mapped. The results indicated that feasibility studies with synthetic modelling are recommended. In practice, forwardmodelling simplification is needed to estimate the resolution; especially, for adaptation of acoustic inversion AI method, widely used for investigation of hydrocarbons, to a new target as recognition of inhomogeneities of salt deposits.

\section{ACKNOWLEDGEMENT}

The work was supported by Geophysics Department AGH UST project (no. 11.11.140.769).

\section{REFERENCES}

[1] Berteussen K.A., Ursin B., Approximate computation of the acoustic impedance from seismic data, Geophysics, 1983, Vol. 48, 10.

[2] KaWALEC-LATALA E., Influency of the level of nosie on the resolution of pseudoimpedance section in NW part of LGOM, Górnictwo Odkrywkowe, 7/2007, 81-86

[3] Kawalec-Latala E., Detection of salts deposits geometry variation, AGH Journal of Mining and Geoengineering, 2012, Vol. 36, No. 2, 161-169.

[4] KaWAlEC-Latala E., The influence of seismic wavelet on the resolution of pseudoimpedance section for construction of underground storage, Minerals Resources Management, 2008, t. 24, z. 2/3, 387-397.

[5] KAWALEC E., Seismic inversion of salt deposits inhomogeneities recognition for the construction of underground reservoirs, Wydawnictwa AGH, Kraków 2009.

[6] KRYNICKI T., Elastic feature of Zechstein rocks, Kwart. Geol., 1980, t. 24, No. 3.

[7] Markiewicz A., BANASZAK A., History of geological surveys of "Kazimierzów" - the oldest rock-salt deposit ("Sieroszowice I" mining area), Czasopismo Naukowo-Techniczne Górnictwo Odkrywkowe, Wrocław, 2005, Vol. XLVII, No. 2, 15-21.

[8] Mascimento T.M., Menezes P.L.T., Braga I.L., High resolution acoustic impedance inversion to characterize turbidities at Marlim Field, Campos Basin, Brasil, Interpretation, 2014, Vol. 2, No. 3, (August 2014), T143-T153.

[9] Oldenburg D.M., Shauer T., LeVy S., Recovery of the acoustic impedance from reflection seismograms, Geophysics, 1983, Vol. 48, 10.

[10] Robinson E.A., Seismic Inversion and Deconvolution, Geophysika Press, London-Amsterdam 1984.

[11] Veeken P.C.H., Da Silva M., Seismic inversion methods and some of their constraints, First break, 2004, Vol. 22, 47-70.

[12] Wiggins R., A. Minimum Entropy Deconvolution, Geoexploration, 1978, Vol. 16, 21-35. 